\title{
Where are Compton-thick radio galaxies? A hard X-ray view of three candidates
}

\author{
F. Ursini,${ }^{1}{ }^{\star}$ L. Bassani, ${ }^{1}$ F. Panessa,${ }^{2}$ A. Bazzano, ${ }^{2}$ A. J. Bird, ${ }^{3}$ A. Malizia, ${ }^{1}$ \\ and P. Ubertini ${ }^{2}$ \\ ${ }^{1}$ INAF-IASF Bologna, Via Gobetti 101, I-40129 Bologna, Italy. \\ ${ }^{2}$ INAF/Istituto di Astrofisica e Planetologia Spaziali, via Fosso del Cavaliere, 00133 Roma, Italy. \\ ${ }^{3}$ School of Physics and Astronomy, University of Southampton, SO17 1BJ, UK.
}

Released Xxxx Xxxxx XX

\begin{abstract}
We present a broad-band X-ray spectral analysis of the radio-loud active galactic nuclei NGC 612, 4C 73.08 and 3C 452, exploiting archival data from NuSTAR, XMM-Newton, Swift and INTEGRAL. These Compton-thick candidates are the most absorbed sources among the hard X-ray selected radio galaxies studied in Panessa et al. (2016). We find an X-ray absorbing column density in every case below $1.5 \times 10^{24} \mathrm{~cm}^{-2}$, and no evidence for a strong reflection continuum or iron $\mathrm{K} \alpha$ line. Therefore, none of these sources is properly Compton-thick. We review other Compton-thick radio galaxies reported in the literature, arguing that we currently lack strong evidences for heavily absorbed radio-loud AGNs.
\end{abstract}

Key words: galaxies: active - galaxies: Seyfert - X-rays: galaxies - X-rays: individual: NGC 612, 4C 73.08, 3C 452

\section{INTRODUCTION}

A significant fraction of active galactic nuclei (AGNs) are known to be intrinsically obscured by gas and dust surrounding their central engine. According to the unified model, the presence of a torus at pc scales can explain the properties of obscured AGNs and the dichotomy with unabsorbed sources, simply due to a different orientation with respect to the line of sight (e.g. Antonucci \& Miller 1985; Antonucci 1993).

Obscured AGNs are considered an important class of sources for two main reasons. First of all, most AGNs in the local universe are absorbed $\left(N_{\mathrm{H}}>10^{22} \mathrm{~cm}^{-2}\right.$; e.g. Risaliti et al. 1999; Bassani et al. 1999). As shown by different X-ray surveys, the observed fraction of absorbed AGNs generally ranges between 40 and 60 per cent (e.g. Burlon et al. 2011, and references therein). However, their intrinsic fraction, i.e. corrected for selection biases, is around 80 per cent (e.g. Malizia et al. 2012). Moreover, obscured AGNs are an important ingredient of both the cosmic X-ray background (e.g. Fabian \& Iwasawa 1999; Gilli et al. 2007) and of the infrared background, where most of the absorbed high-energy radiation is re-emitted (e.g. Risaliti et al. 2002).

In particular, Seyfert 2 galaxies in which the absorbing material has $N_{\mathrm{H}} \geqslant \sigma_{\mathrm{T}}^{-1}=1.5 \times 10^{24} \mathrm{~cm}^{-2}$, where $\sigma_{\mathrm{T}}$ is the Thomson cross-section, are called Compton-thick (CT). Such high column densities produce a photoelectric cut-off at energies of $10 \mathrm{keV}$ or more. Hard X-rays data are thus needed to properly study CT sources (see, e.g., the analysis of BeppoSAX data by Matt et al.

* e-mail: ursini@iasfbo.inaf.it
2000). A number of local CT AGNs have been detected thanks to recent hard X-ray surveys with INTEGRAL (Sazonov et al. 2008; Malizia et al. 2009, 2012) and Swift (e.g. Burlon et al. 2011; Ricci et al. 2015). At high redshift, the rest-frame hard X-ray emission becomes observable below $10 \mathrm{keV}$ and CT AGNs have been detected using deep surveys by XMM-Newton (e.g. Lanzuisi et al. 2015) and Chandra (e.g. Brightman et al. 2014). While the observed fraction of CT AGNs is generally found to be of a few percent, their intrinsic fraction could be as high as 20-30 per cent. This estimate remains highly uncertain, owing to the difficulties in detecting and classifying such heavily obscured sources.

It is a matter of debate whether differences exist in the absorption properties between different classes of AGNs, depending on their physical parameters (like the accretion rate or the luminosity, e.g. Elitzur \& Shlosman 2006). For example, the fraction of obscured AGN is observed to decrease with increasing X-ray luminosity (e.g. Ueda et al. 2003; La Franca et al. 2005; Sazonov et al. 2015), although with some uncertainties related to luminous but heavily obscured sources (Mateos et al. 2017), and possibly with decreasing redshift (e.g. La Franca et al. 2005). Radio galaxies are particularly interesting in this respect, because the presence of a jet could have an impact on the circumnuclear environment and on the obscuring medium. Large fractions of X-ray obscured radio galaxies are generally found (e.g. Sambruna et al. 1999; Evans et al. 2006; Hardcastle et al. 2009; Wilkes et al. 2013). In particular, Wilkes et al. (2013) reported a fraction of 20 per cent of CT sources in a high-redshift sample $(1<z<2)$. However, only a few radio galaxies in the local Universe have been reported as CT: Mrk 668 (Guainazzi et al. 2004), 3C 284 (Hardcastle et al. 2006), 
Table 1. Basic data of the three sources selected from P16.

\begin{tabular}{lccc}
\hline \hline & NGC 612 & 4C 73.08 & 3C 452 \\
\hline Optical classification & Sy2 & Sy2 & Sy2 \\
Radio morphology & FRI/II & FRII & FRII \\
Host galaxy type & S0 & E & E \\
Redshift & 0.029771 & 0.058100 & 0.081100 \\
Galactic $N_{\mathrm{H}}\left(10^{20} \mathrm{~cm}^{-2}\right)$ & 1.85 & 2.41 & 11.9 \\
\hline
\end{tabular}

3C 223 (LaMassa et al. 2014; Corral et al. 2014), 3C 321 (Evans et al. 2008; Severgnini et al. 2012), 3C 452 (Fioretti et al. 2013), TXS 2021+614 (Siemiginowska et al. 2016) and PKS 1607+26 (Tengstrand et al. 2009; Siemiginowska et al. 2016).

In a recent work, Panessa et al. (2016, P16 hereafter) investigated the role of absorption in radio galaxies. The sample analysed by P16 consisted of 64 AGNs showing extended radio emission, selected from hard X-ray catalogues by Bassani et al. (2016). Being hard X-ray selected, this sample is relatively unbiased towards absorption, at least up to a few $\times 10^{24} \mathrm{~cm}^{-2}$. P16 derived the column density distribution, and found an observed fraction of absorbed sources of 40 per cent ( 75 per cent among the type 2 s). However, the fraction of CT sources was found to be no more than 2-3 per cent, whereas 7-10 per cent would have been expected (e.g. Malizia et al. 2012).

In this paper, we focus on the most absorbed radio galaxies reported in P16, with the aim of studying their broad-band X-ray spectra and to well constrain their column density. We take advantage of archival NUSTAR data, which are very helpful in constraining the spectral shape of absorbed sources thanks to the high-energy coverage (e.g. Baloković et al. 2014). The structure of the paper is as follows. In Section 2 we present the sources and their archival $\mathrm{X}$-ray data used. In Section 3, we present the spectral analysis. We discuss the results in Section 4, and summarize the conclusions in Section 5 .

\section{OBSERVATIONS AND DATA REDUCTION}

We selected the two sources that, according to the results of P16, show an $N_{\mathrm{H}}$ consistent with being larger than $10^{24} \mathrm{~cm}^{-2}$. These are NGC 612 and 4C 73.08 (VII Zw 292), for which P16 reported $\log N_{\mathrm{H}}=24.02 \pm 0.2$ and $23.96_{-0.16}^{+0.20}$, respectively. We also included $3 \mathrm{C} 452\left(\log N_{\mathrm{H}}=23.77_{-0.07}^{+0.06}\right.$ according to P16) which has been labelled as a CT candidate by Fioretti et al. (2013).

\subsection{The sources}

The basic data of NGC 612, 4C 73.08 and 3C 452 are reported in Table 1.

NGC 612 is a rare case of a radio source hosted by a spiral galaxy (e.g. Véron-Cetty \& Véron 2001), with a prominent dust lane along the disc (e.g. Ekers et al. 1978; Véron-Cetty \& Véron 2001). The optical spectrum is consistent with the presence of a reddened young stellar population of age $<0.1 \mathrm{Gyr}$ (Holt et al. 2007). The infrared properties indicate an active star formation taking place in a warped disc (Duah Asabere et al. 2016). The radio morphology is also peculiar, as it shows an eastern radio lobe of type FRII and a western lobe of type FRI (Gopal-Krishna \& Wiita 2000). Furthermore, NGC 612 is surrounded by a $140 \mathrm{kpc}-$ wide disc of neutral hydrogen, and an $\mathrm{H}_{\mathrm{I}}$ bridge is seen towards the neighbour galaxy NGC 619 (Emonts et al. 2008). The AGN in NGC 612 is optically classified as a Seyfert 2 (e.g. Véron-Cetty \& Véron 2006; Parisi et al. 2009) and it is absorbed in the X-rays. Using Suzaku data, Eguchi et al. (2009) found $N_{\mathrm{H}} \simeq 1.1 \times 10^{24} \mathrm{~cm}^{-2}$ and suggested a scenario where the $\mathrm{X}$-ray source is obscured by a torus with a half-opening angle of 60-70 deg, seen from a nearly edge-on angle.

4C 73.08 is a giant radio galaxy, with a linear size of around 1 Mpc (e.g. Bassani et al. 2016). It exhibits a complex morphology, but overall consistent with a FRII (e.g. Lara et al. 2001; Strom et al. 2013). The AGN is optically classified as a Seyfert 2 (e.g. Hewitt \& Burbidge 1991; Parisi et al. 2014). This source is poorly studied in the X-rays, however Evans et al. (2008) inferred an absorbing column density of around $9 \times 10^{23} \mathrm{~cm}^{-2}$ for the nucleus, from the analysis of XMM-Newton data.

$3 \mathrm{C} 452$ is a radio galaxy with a symmetrical FRII morphology (e.g. Black et al. 1992), optically classified as a Seyfert 2 (e.g. Véron-Cetty \& Véron 2006). From Chandra data, Isobe et al. (2002) found the nucleus to be absorbed by a column density of around $6 \times 10^{23} \mathrm{~cm}^{-2}$, and detected a diffuse X-ray emission from the lobes. Similar results were obtained by Evans et al. (2006) with the same data set. Using XMM-Newton data, Shelton et al. (2011) performed a detailed X-ray study of both the AGN and the lobes, constraining their inverse Compton emission and environmental impact. No significant variations were found from the comparison with the Chandra observation, while the absorbing column density was found to be around $4 \times 10^{23} \mathrm{~cm}^{-2}$ for the AGN. The same column density was reported by Fioretti et al. (2013), who analysed Suzaku data. Finally, according to the results of Fioretti et al. (2013), the hard energy band of the X-ray spectrum is dominated by Compton reflection off cold matter, with a reflection fraction (as defined in Magdziarz \& Zdziarski 1995) found to be $R>400$. This unphysical value could be due to an inhomogeneous circumnuclear material, where a significant fraction of the solid angle is covered by a gas thicker than that along the line of sight (Fioretti et al. 2013).

\subsection{The X-ray data}

For the three sources we analysed archival NuSTAR data. Being sensitive in the 3-79 keV band, NUSTAR (Harrison et al. 2013) allows us to constrain both the spectral shape at high energies and the absorbing column density. We also included the average 70-month Swift/BAT spectra (Baumgartner et al. 2013) ${ }^{1}$ to extend the coverage up to $195 \mathrm{keV}$, and INTEGRAL/IBIS data (Ubertini et al. 2003) for 3C 452. Moreover, to better constrain the column density, we included the soft X-ray spectra by XMM-Newton (Jansen et al. 2001) when possible, namely in the case of NGC 612 and 4C 73.08.

We report in Table 2 the logs of the archival NuSTAR data sets of the three sources. We reduced the NUSTAR data using the standard pipeline (NUPIPELINE) in the NUSTAR Data Analysis Software (NUSTARDAS, v1.8.0; part of the HEASOFT distribution as of version 6.22), using calibration files from NuSTAR CALDB v20170817. We extracted the spectra using the standard tool NUPRODUcTs for each of the two hard X-ray detectors, which reside in the corresponding focal plane modules A and B (FPMA and FPMB). We extracted the source data from circular regions with a radius of $75 \mathrm{arcsec}$, and the background from a blank area close to the source. The spectra were

\footnotetext{
${ }^{1}$ https://swift.gsfc.nasa.gov/results/bs70mon/
} 
Table 2. Logs of the NUSTAR observations of the sources.

\begin{tabular}{cccc}
\hline \hline Source & Obs. Id. & $\begin{array}{c}\text { Start time (UTC) } \\
\text { yyyy-mm-dd }\end{array}$ & $\begin{array}{c}\text { Net exp. } \\
\text { (ks) }\end{array}$ \\
\hline NGC 612 & 60061014002 & $2012-09-14$ & 17 \\
\hline 4C 73.08 & 61060374002 & $2016-12-05$ & 13 \\
\hline 3C 452 & 60261004002 & $2017-05-01$ & 52 \\
\hline
\end{tabular}

binned to have a signal-to-noise ratio larger than 3 in each spectral channel, and not to oversample the instrumental resolution by a factor greater than 2.5. The spectra from the two detectors were analysed jointly, but not combined.

The XMM-Newton data were processed using the $X M M$ Newton Science Analysis System (sas v16). We used the EPIC-pn data, because of the much larger effective area of pn than that of the MOS detectors. The source extraction radii and screening for highbackground intervals were determined through an iterative process that maximizes the signal-to-noise ratio (see Piconcelli et al. 2004). We extracted the background from circular regions with a radius of 50 arcsec, while the source extraction radii were in the range $20-40$ arcsec. We binned the spectra to have at least 30 counts per spectral bin, and not oversampling the instrumental resolution by a factor larger than 3 .

The INTEGRAL spectrum of 3C 452 consists of ISGRI data from several pointings between revolution 12 and 530 (from the fourth IBIS catalogue; Bird et al. 2010). The data extraction was carried out following the procedure described in Molina et al. (2013).

\section{SPECTRAL ANALYSIS}

The spectral analysis was carried out with the XSPEC 12.9.1 package (Arnaud 1996), using the $\chi^{2}$ minimisation technique. All errors are quoted at the 90 per cent confidence level. As we explain below, we fitted the 3-79 keV NuSTAR spectra and the 14-195 keV Swift/BAT spectra simultaneously, leaving the cross-calibration constants free to vary, after checking for consistency. In the case of NGC 612 and 4C 73.08, we included XMM-Newton/pn data to extend the broadband analysis down to $0.3 \mathrm{keV}$. We did not include XMM-Newton data of 3C 452, because the pn spectrum was not consistent with the NUSTAR one (see Sect. 3.3). Finally, in the case of 3C 452 we exploited INTEGRAL/IBIS data.

In all cases, we first fitted the data with a phenomenological model including an absorbed power law. Then, we tested a more physical absorption model, i.e. a gas torus (мYTORus: Murphy \& Yaqoob 2009). We always included Galactic absorption, fixing the hydrogen column densities to the values obtained from the H I map of Kalberla et al. (2005, as given by the tool $\mathrm{NH}$ in the HEASoFT package). For all models, we adopted the chemical abundances of Anders \& Grevesse (1989) and the photoelectric absorption crosssections of Verner et al. (1996).

In Fig. 1, we plot for comparison the NUSTAR spectra of the three sources with the best-fitting power law above $10 \mathrm{keV}$ (with tied parameters), showing the effects of absorption at lower energies.

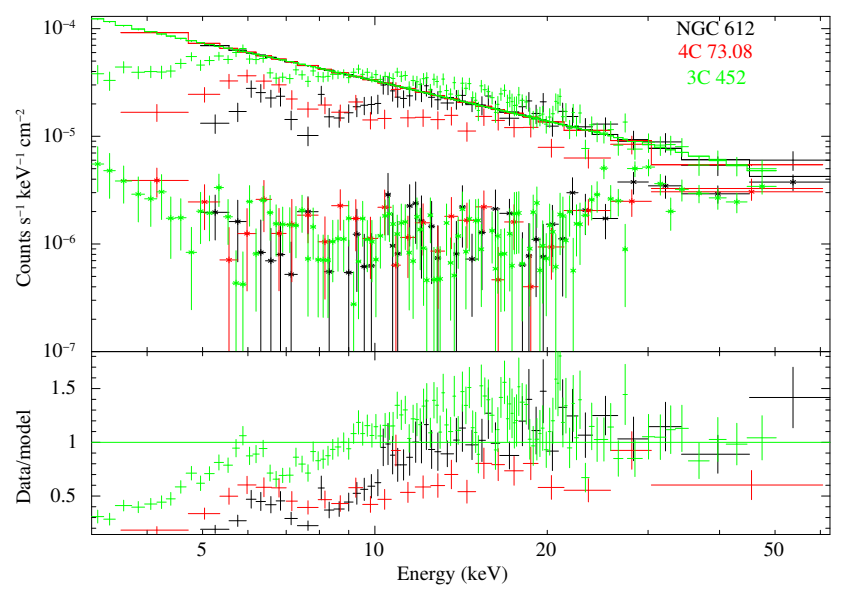

Figure 1. Upper panel: NUSTAR/FPMA spectra of NGC 612 (black), 4C 73.08 (red) and 3C 452 (green) plotted with the corresponding background level. Lower panel: residuals when fitting simultaneously the spectra above $10 \mathrm{keV}$ with a simple power law. The data were binned for plotting purposes.

\subsection{NGC 612}

NUSTAR observed this source in 2012 with a net exposure of $17 \mathrm{ks}$, as part of the NUSTAR serendipitous survey (Lansbury et al. 2017). XMM-Newton observed the source in 2006 (Obs. Id. 0312190201 ), with a net exposure of $10 \mathrm{ks}$. Before using the XMM-Newton/pn data, we checked the flux variation of the source between the XMMNewton and NuSTAR observations. We thus fitted the NuSTAR spectrum with a simple power law modified by photoelectric absorption (ZPHABS model in XSPEC) to derive the $3-10 \mathrm{keV}$ flux, which was found to be $1.15 \pm 0.08 \times 10^{-12} \mathrm{erg} \mathrm{cm}^{-2} \mathrm{~s}^{-1}$. The photon index was $\Gamma=1.60 \pm 0.16$ and the column density $N_{\mathrm{H}}=84 \pm 12 \times 10^{22}$ $\mathrm{cm}^{-2}$. Next, we fitted the XMM-Newton/pn spectrum in the 3-10 $\mathrm{keV}$ band with the same model with fixed parameters, only allowing for flux variability. The $3-10 \mathrm{keV}$ flux measured by pn was $1.66 \pm 0.11 \times 10^{-12} \mathrm{erg} \mathrm{cm} \mathrm{cm}^{-2} \mathrm{~s}^{-1}$. The flux variation is thus of $30-50$ per cent. However, given the large error bars and the crosscalibration uncertainties of 10 per cent between NUSTAR and XMMNewton/pn (Madsen et al. 2017), we did not consider it a dramatic variation and we fitted the two spectra simultaneously, allowing for a scaling factor between pn and NUSTAR. We verified a posteriori that the spectral parameters are consistent with those measured by NUSTAR alone (see Table 3). We also included the 70-month Swift/BAT spectrum, which is consistent with NuSTAR with a crossnormalization constant of $1.25 \pm 0.13$.

As a first step, we fitted the NuSTAR, XMM-Newton/pn and Swift/BAT spectra in the $0.3-195 \mathrm{keV}$ range, with a phenomenological model including a power law multiplied by zPHABs ("ZPHABS model"). To find a good fit to the pn data, we needed to include further emission components that were not absorbed by ZPHABS, i.e. not originating from the central X-ray source. This "soft excess" above the absorbed power law is ubiquitous in Seyfert $2 \mathrm{~s}$ (e.g. Turner et al. 1997; Guainazzi et al. 2005), and can be due to scattering of the primary continuum in an optically thin region (e.g. Turner et al. 1997; Ueda et al. 2007) and/or photoionization of circumnuclear gas (e.g. Guainazzi \& Bianchi 2007). For NGC 612, we included: (i) a secondary power law, with the parameters tied to those of the primary one, to model optically-thin scattered continuum; we added a free multiplicative constant corresponding to the 
scattered fraction $f_{s}$, expecting $f_{s}$ to be of the order of a few percent or less (e.g. Turner et al. 1997); (ii) a thermal component (APEC in XSPEC), to model photoionized emission. We allowed for free cross-normalization constants between NuSTAR/FPMA and FPMB $\left(K_{A-B}\right)$, pn $\left(K_{A-p n}\right)$ and BAT $\left(K_{A-B A T}\right)$. In XSPEC notation, the model reads: CONST*PHABS*(ZPHABS*POWERLAW + POWERLAW + APEC).

The results are summarized in Table 3. We find a good fit $\left(\chi^{2} /\right.$ dof $\left.=131 / 132\right)$ and constraints on the photon index and column density. We tested the presence of an exponential cut-off at high energies, but this parameter was unconstrained. We also tested the presence of a $\mathrm{Fe} \mathrm{K} \alpha$ emission line at $6.4 \mathrm{keV}$, which is a common feature in the X-ray spectra of obscured AGNs (e.g. Guainazzi et al. 2005), and which can be particularly prominent in CT sources dominated by reflection (e.g. Matt et al. 1996). Including a narrow Gaussian line, we found a rest-frame energy of $6.31 \pm 0.08$ $\mathrm{keV}$ and a line flux of $2.6 \pm 1.6 \times 10^{-6}$ photons $\mathrm{cm}^{-2} \mathrm{~s}^{-1}$. The fit is improved, albeit not dramatically $\left(\chi^{2} /\right.$ dof $=124 / 130$, i.e. $\Delta \chi^{2} / \Delta$ dof $=-7 /-2$, with a probability of chance improvement of 0.03 calculated with an $F$-test). The equivalent width of the line is $110_{-60}^{+80} \mathrm{eV}$. Then, we tested the presence of a Comptonreflected component by replacing the power law with the PEXRAV model (Magdziarz \& Zdziarski 1995). This model includes the continuum reflected off an infinite slab of infinite optical depth, which can be considered an approximation for the reflection off a finitedensity torus. We fixed the inclination angle at $60 \mathrm{deg}$, and we assumed solar abundances. However, the fit is not significantly improved, and the reflection fraction is poorly constrained (we only derive an upper limit of 1.6).

Next, we tested a more physical absorption model by a torus. We replaced the zPHABs component with the MYTORUs model, which describes absorption with self-consistent Compton reflection and iron fluorescent lines from a gas torus with a half-opening angle of 60 deg (Murphy \& Yaqoob 2009; Yaqoob 2012). In this case, we had to use pn data above $0.5 \mathrm{keV}$, because this is the lower bound for which мYтоRUs is valid. The inclination angle of the torus was fixed at $90 \mathrm{deg}$, with no improvement by leaving it free to vary. We included the scattered and line emission components, linking their column densities to that of the absorber ("coupled" model). The photon indexes and normalizations of the scattered and line components were tied to those of the primary power law, allowing for a free relative normalization by means of a multiplicative constant ${ }^{2}$. We assumed the standard мYтоRUs configuration $A_{S}=A_{L}$, where $A_{S}$ is the scaling factor for the scattered component and $A_{L}$ that of the line component (Yaqoob 2012). In XSPEC notation, the model reads: CONST $1{ }^{*}$ PHABS $*$ (MYTORUSZ* ${ }^{*}$ POWERLAW + APEC + POWERLAW + CONST $2 *$ MYTORUSS + CONST $2 *$ MYTORUSL).

The results for the mYTORus model are reported in Table 3 (see also the contours in Fig. 3), while in Fig. 2 we show the data, residuals and best-fitting model. The column density is $94 \pm 7 \times 10^{22}$ $\mathrm{cm}^{-2}$. The parameters are essentially consistent with those of the ZPHABs model, and the fit is slightly improved $\left(\chi^{2} /\right.$ dof $=121 / 129$ i.e. $\Delta \chi^{2} / \Delta$ dof $=-10 /-3$, but we have 2 dof less because of the different energy range). The parameter $A_{S}$ is $0.25_{-0.19}^{+0.22}$, i.e. relatively low, indicating a weak Compton-scattered continuum. To further test the significance of this result, we fixed $A_{S}$ to unity, finding a worse fit $\left(\chi^{2} /\right.$ dof $=142 / 130$ i.e. $\Delta \chi^{2} / \Delta$ dof $\left.=+21 /+1\right)$. We note that $A_{S}=1$ would correspond to the default, steady-state configu-

2 The fluxes (or equivalent widths) of the fluorescence lines are not free parameters in MYTORUs, because they are determined by the incident continuum (for a detailed explanation, see Chapter 7 of the MYTORus manual.

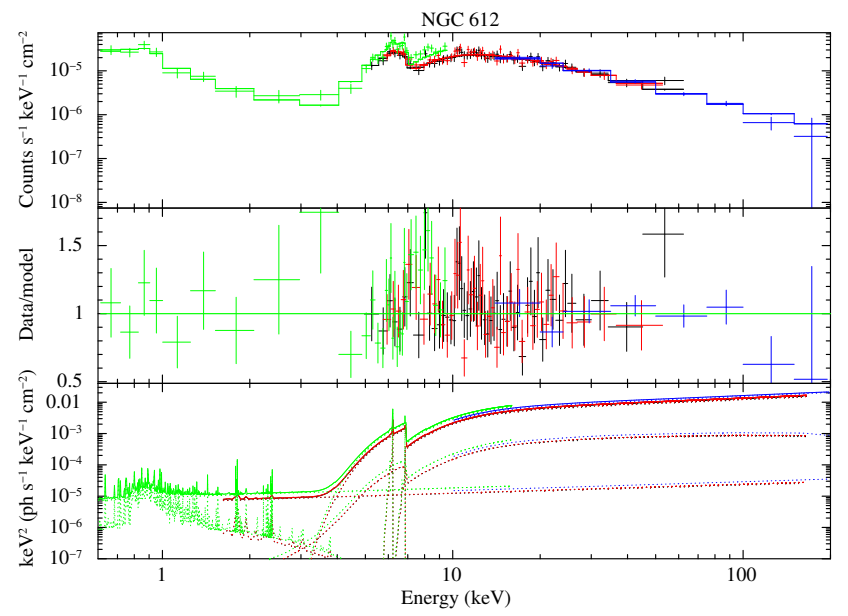

Figure 2. Broad-band X-ray data and best-fitting MYTORUs model for NGC 612 (see Table 3). Upper panel: spectra and folded model from NuSTAR/FPMA (black), FPMB (red), XMM-Newton/pn (green), Swift/BAT (blue). Middle panel: ratio of data to model. Lower panel: best-fitting model $E^{2} f(E)$, consisting of the absorbed primary power law, the scattered power law, the MYTORUs reflection component and the thermal component APEC.

ration of MYTORUs, in which the torus has a covering factor of 0.5 (Murphy \& Yaqoob 2009). However, $A_{S}$ cannot be immediately related to the covering factor, because it is a simple scalar, while the precise shape of the scattered continuum depends on several parameters, such as the geometry of the system, elemental abundances and putative time delays between the scattered and intrinsic continuum (e.g. Yaqoob 2012). Anyway, the case $A_{S}=0$ would correspond to a geometry in which the torus subtends a small solid angle, so that the scattered continuum and the fluorescence emission are negligible. In this case, the MYTORUs model simply consists in photoelectric absorption plus additional attenuation due to Compton scattering. This last effect is not included in models like ZPHABS, and this is the reason why the normalization of the primary power law, as well as the estimated intrinsic luminosity, is larger using MYTORUS.

\section{$3.2 \quad 4 \mathrm{C} 73.08$}

This source was observed by NUSTAR in 2016 with a net exposure of $13 \mathrm{ks}$, and by XMM-Newton in 2007 (Obs. Id. 0404050601) with a net exposure of $7.5 \mathrm{ks}$. As for NGC 612, we first fitted the $N u S$ $T A R$ data with a power law modified by zPHABs, finding a $3-10 \mathrm{keV}$ flux of $1.5 \pm 0.1 \times 10^{-12} \mathrm{erg} \mathrm{cm}^{-2} \mathrm{~s}^{-1}$. The photon index was found to be $1.4 \pm 0.2$, and the column density $40 \pm 10 \times 10^{22} \mathrm{~cm}^{-2}$. Applying this model to the XMM-Newton/pn data in the 3-10 keV band, we found a flux of $1.05 \pm 0.15 \times 10^{-12} \mathrm{erg} \mathrm{cm}^{-2} \mathrm{~s}^{-1}$. The flux variation is thus of around 50 per cent. Nevertheless, we fitted the pn data jointly with NUSTAR, allowing for a free scaling factor and verifying that the spectral parameters were consistent with those measured by NuSTAR. Finally, we included the 70-month Swift/BAT spectrum, finding a cross-normalization constant of $0.76 \pm 0.20$ with respect to NUSTAR.

Like for NGC 612, we first fitted the NuSTAR, XMMNewton/pn and Swift/BAT spectra in the $0.3-195 \mathrm{keV}$ range using the zPHABS model. In the case of $4 \mathrm{C} 73.08$, however, we fitted the "soft excess" above the absorbed power law with a sec- 


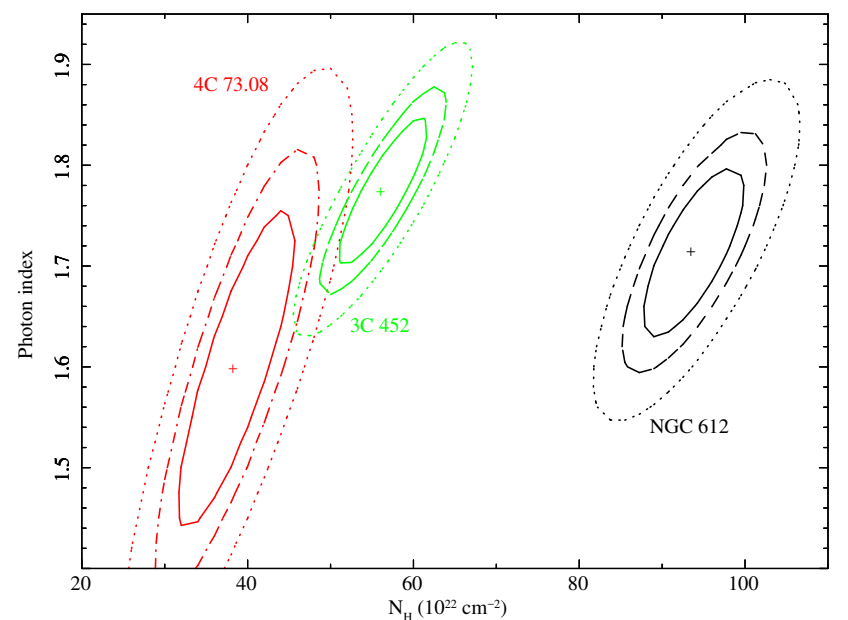

Figure 3. Contour plots of the column density $N_{\mathrm{H}}$ versus the photon index of the primary power law (мYToRus model, see Table 3) for NGC 612 (black), 4C 73.08 (red) and 3C 452 (green). Solid, dashed and dotted lines correspond to 68 per cent, 90 per cent and 99 per cent confidence levels, respectively.

ondary power law only, as a thermal component was not needed. The results are summarized in Table 3 . The fit is statistically good $\left(\chi^{2} /\right.$ dof $\left.=145 / 152\right)$ and, despite the larger error bars compared with NGC 612, the column density is constrained and below $10^{24}$ $\mathrm{cm}^{-2}$. We found no constraint on the high-energy cut-off, and a hint of a narrow Fe $\mathrm{K} \alpha$ emission line with rest-frame energy of $6.45 \pm 0.15 \mathrm{keV}$, line flux of $4 \pm 3 \times 10^{-6}$ photons $\mathrm{cm}^{-2} \mathrm{~s}^{-1}$ and equivalent width of $120 \pm 100 \mathrm{eV}\left(\chi^{2} / \mathrm{dof}=142 / 150\right.$, i.e. $\Delta \chi^{2} / \Delta$ dof $=-3 /-2$, with a probability of chance improvement of 0.2). Then, we tested the presence of a Compton-reflected component with PEXRAV, finding no major improvement and a poor constraint on the reflection fraction (the upper limit was 2.2).

Finally, we tested the MYToRus model (see Table 3 and the contours in Fig. 3) using the same standard setting as for NGC 612. The best-fitting parameters are consistent within the errors with those of the zPHABS model, and the fit is slightly improved $\left(\chi^{2} /\right.$ dof $=$ $140 / 151$ i.e. $\Delta \chi^{2} / \Delta$ dof $\left.=-5 /-1\right)$. In particular, the column density is $39 \pm 8 \times 10^{22} \mathrm{~cm}^{-2}$. The parameter $A_{S}$ is not well constrained, as we find only an upper limit of 2.4. The data, residuals and bestfitting model are shown in Fig. 4.

\section{$3.3 \quad 3 C 452$}

NUSTAR observed this source in 2017 with a net exposure of $52 \mathrm{ks}$, as part of the NUSTAR extragalactic survey. We fitted the NUSTAR spectrum jointly with the 70-month Swift/BAT spectrum, which had a cross-normalization constant of $0.9 \pm 0.1$ with respect to NuSTAR. 3C 452 was also observed by INTEGRAL/IBIS with an exposure of $100 \mathrm{ks}$, and is part of the fourth INTEGRAL/IBIS catalogue (Bird et al. 2010; Malizia et al. 2012). We thus included the IBIS spectrum in the $20-150 \mathrm{keV}$ range, finding a cross-normalization constant of $1.3 \pm 0.5$ with respect to NuSTAR. In the case of $3 \mathrm{C} 452$, we did not use soft X-ray data. Although the source was observed by XMM-Newton in 2008 and by Suzaku in 2007, the corresponding soft X-ray spectra are not fully consistent with NuSTAR. Indeed, for NuSTAR we found a photon index of $1.76 \pm 0.07$, while Shelton et al. (2011) reported a photon index of $1.26_{-0.27}^{+0.32}$ for $X M M$ -

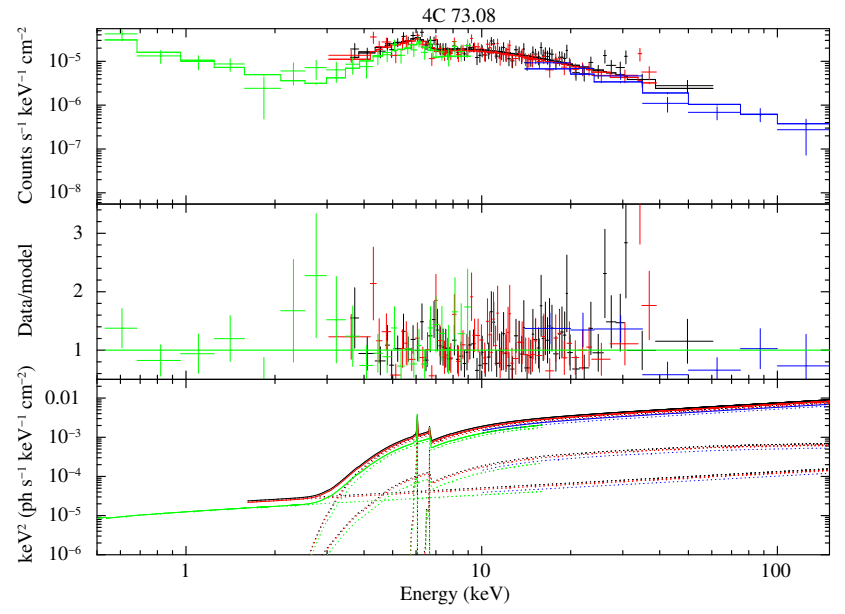

Figure 4. Broad-band X-ray data and best-fitting MYTORUs model for 4C 73.08. Upper panel: spectra and folded model from NuSTAR/FPMA (black), FPMB (red), XMM-Newton/pn (green), Swift/BAT (blue). Middle panel: ratio of data to model. Lower panel: best-fitting model $E^{2} f(E)$, consisting of the absorbed primary power law, the scattered component and the MYTORUS reflection component (which, however, is consistent with zero).

Newton/pn, and Fioretti et al. (2013) reported $1.55_{-0.11}^{+0.14}$ for Suzaku. However, the NuSTAR+Swift/BAT+INTEGRAL data set was sufficient to yield good constraints on both the photon index and the column density.

Also in this case, we first fitted the spectra with a power law modified by zPHABs. However, the fit was relatively poor $\left(\chi^{2} /\right.$ dof $=$ $276 / 243$ ) with significant residuals in the $3-5 \mathrm{keV}$ band. We found a much better fit $\left(\chi^{2} /\right.$ dof $=220 / 242$, i.e. $\Delta \chi^{2} / \Delta$ dof $\left.=-56 /-1\right)$ by including a secondary power law, with a scattered fraction $f_{s}=0.09 \pm 0.01$. Part of this excess could be actually due to extended emission related to the radio structure (Isobe et al. 2002; Fioretti et al. 2013). However, this contamination is relatively small above $3 \mathrm{keV}$ and we do not expect it to affect the measurement of the column density. The best-fitting parameters are reported in Table 3. We did not find any statistical improvement of the fit by adding a narrow $\mathrm{Fe} \mathrm{K} \alpha$ line at $6.4 \mathrm{keV}$ (the equivalent width being less than $50 \mathrm{eV}$ ), nor by adding a reflection component with PEXRAV, with an upper limit on the reflection fraction of 0.8. Including an exponential high-energy cut-off results in a minor improvement of the fit $\left(\chi^{2} /\right.$ dof $=215 / 241$, i.e. $\Delta \chi^{2} / \Delta$ dof $=-5 /-1$ and a probability of chance improvement of 0.02). Formally, the cut-off energy was found to be $120_{-55}^{+360} \mathrm{keV}$ within the 90 per cent confidence level. To further explore this possibility, we replaced the simple power law with a thermal Comptonization model, namely NTHсOMP (Zdziarski et al. 1996; Życki et al. 1999). We assumed illumination from a disc blackbody with a seed photon temperature of $10 \mathrm{eV}$. We found a nearly equivalent fit $\left(\chi^{2} / \mathrm{dof}=218 / 241 ; \Delta \chi^{2}=+3\right)$, with $\Gamma=1.75 \pm 0.06$ and only a lower limit on the electron temperature of $30 \mathrm{keV}$.

Then, we tested the мYтоRUs model with the standard setting (see Table 3 and Fig. 3). In this case, we found no improvement relative to the initial zPHABs fit $\left(\chi^{2} /\right.$ dof $=223 / 241$, i.e. $\Delta \chi^{2} / \Delta$ dof $\left.=+3 /-1\right)$. The column density is $57 \pm 6 \times 10^{22} \mathrm{~cm}^{-2}$, and the parameter $A_{S}$ is less than 0.2 (fixing $A_{S}$ to unity gives a worse fit with $\chi^{2} /$ dof $=250 / 242$, i.e. $\Delta \chi^{2} / \Delta$ dof $\left.=+27 /+1\right)$. The data, residuals and best-fitting model are shown in Fig. 5 . 


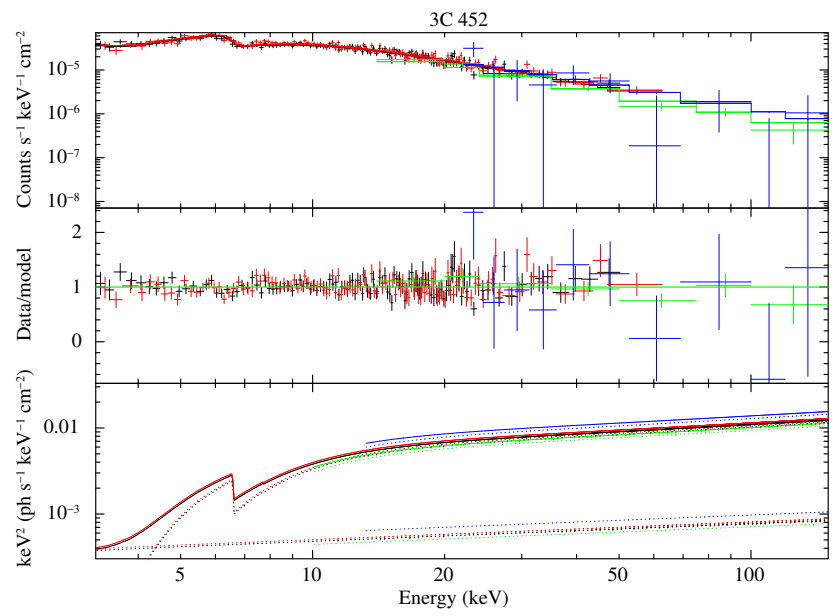

Figure 5. Broad-band X-ray data and best-fitting model for 3C 452. Upper panel: spectra and folded model from NuSTAR/FPMA (black), FPMB (red), Swift/BAT (green), INTEGRAL/IBIS (blue). Middle panel: ratio of data to model. Lower panel: best-fitting model $E^{2} f(E)$, consisting of the absorbed primary power law and the scattered component. Only the absorption component of MYTORUs is needed, while the reflection component is not significant.

Table 3. Best-fitting parameters of the baseline model (zPHABs model) and of the MYTORUs model.

\begin{tabular}{|c|c|c|c|}
\hline & NGC 612 & 4C 73.08 & $3 \mathrm{C} 452$ \\
\hline \multicolumn{4}{|c|}{ ZPHABS model } \\
\hline$\Gamma$ & $1.64^{+0.09}$ & $1.61 \pm 0.17$ & $1.76 \pm 0.07$ \\
\hline$N_{\text {Pow }}\left(10^{-3}\right)$ & $\begin{array}{r}-0.19 \\
2.7 \pm 1.0\end{array}$ & $1.2_{-0.5}^{+0.8}$ & $3.7_{-0.8}^{+1.0}$ \\
\hline$N_{\mathrm{H}}\left(10^{22} \mathrm{~cm}^{-2}\right)$ & $93_{-8}^{+5}$ & $\begin{array}{l}-0.5 \\
40 \pm 8\end{array}$ & $57 \pm 6$ \\
\hline$f_{s}\left(10^{-2}\right)$ & $0.27_{-0.08}^{-8.15}$ & $2 \pm 1$ & $9 \pm 1$ \\
\hline$k T_{\mathrm{APEC}}(\mathrm{keV})$ & $0.86_{-0.19}^{-0.08}$ & - & - \\
\hline$N_{\text {APEC }}\left(10^{-6}\right)$ & $3.2_{-1 .}^{+0.19}$ & - & - \\
\hline$F_{2-10 \mathrm{keV}}\left(10^{-12} \mathrm{erg} \mathrm{cm}^{-2} \mathrm{~s}^{-1}\right)$ & $1.11 \pm 0.07$ & $1.6 \pm 0.1$ & $3.37 \pm 0.09$ \\
\hline$F_{10-100 \mathrm{keV}}\left(10^{-11} \mathrm{erg} \mathrm{cm}^{-2} \mathrm{~s}^{-1}\right)$ & $3.0 \pm 0.2$ & $1.6 \pm 0.2$ & $2.9^{+0.1}$ \\
\hline$L_{2-10 \mathrm{keV}}^{\text {intr }}\left(10^{43} \mathrm{erg} \mathrm{s}^{-1}\right)$ & $2.3 \pm 0.3$ & $4.2 \pm 1.0$ & $19 \pm 2$ \\
\hline$L_{10-100 \mathrm{kVV}}^{\text {intr }}\left(10^{43} \mathrm{erg} \mathrm{cm}^{-2} \mathrm{~s}^{-1}\right)$ & $6.6 \pm 0.5$ & $13 \pm 2$ & $44 \pm 2$ \\
\hline$K_{A-B}$ & $1.02 \pm 0.07$ & $0.92 \pm 0.08$ & $1.04 \pm 0.03$ \\
\hline$K_{A-p n}$ & $1.50 \pm 0.15$ & $0.67 \pm 0.11$ & - \\
\hline$K_{A-B A T}$ & $1.25 \pm 0.13$ & $0.76 \pm 0.20$ & $0.9 \pm 0.1$ \\
\hline$K_{A-I B I S}$ & - & - & $1.3 \pm 0.5$ \\
\hline$\chi^{2} /$ dof & $131 / 132$ & $145 / 152$ & $220 / 242$ \\
\hline \multicolumn{4}{|c|}{ MYTORUs model } \\
\hline$\Gamma$ & $1.71_{-0.05}^{+0.09}$ & $1.60 \pm 0.17$ & $1.79 \pm 0.07$ \\
\hline$N_{\text {Pow }}\left(10^{-3}\right)$ & $6 \pm 2$ & $1.4^{+1.2}$ & $6.4^{+1.0}$ \\
\hline$N_{\mathrm{H}}\left(10^{22} \mathrm{~cm}^{-2}\right)$ & $94 \pm 7$ & $39 \pm 8$ & $57 \pm 6$ \\
\hline$A_{S}=A_{L}$ & $0.25^{+0.22}$ & $<2.4$ & $<0.2$ \\
\hline$f_{s}\left(10^{-2}\right)$ & $0.11_{-0.04}^{+0.19}$ & $1.5_{-08}^{+1.6}$ & $5.4_{-07}^{+1.2}$ \\
\hline$L_{2-1}^{\text {intr }}\left(10^{43} \mathrm{erg} \mathrm{s}^{-1}\right)$ & $4.7 \pm 1.0$ & $5 \pm 1$ & $31 \pm 5$ \\
\hline$L_{10-100 \mathrm{keV}}^{\text {intr }}\left(10^{43} \mathrm{erg} \mathrm{cm}^{-2} \mathrm{~s}^{-1}\right)$ & $12 \pm 1$ & $15 \pm 3$ & $67 \pm 3$ \\
\hline$\chi^{2} /$ dof & $121 / 129$ & $140 / 151$ & $223 / 241$ \\
\hline
\end{tabular}

Table 4. Properties of the neutral, narrow Fe $\mathrm{K} \alpha$ emission line as found from the zPHABs model. For $3 \mathrm{C} 452$, we fixed the energy at $6.4 \mathrm{keV}$ to test the significance of the line in the NUSTAR data.

\begin{tabular}{|c|c|c|c|}
\hline & NGC 612 & 4C 73.08 & $3 \mathrm{C} 452$ \\
\hline Rest-frame energy (keV) & $6.31 \pm 0.08$ & $6.45 \pm 0.15$ & $6.4(\mathrm{f})$ \\
\hline Flux $\left(10^{-6}\right.$ photons $\left.\mathrm{cm}^{-2} \mathrm{~s}^{-1}\right)$ & $2.6 \pm 1.6$ & $4 \pm 3$ & $<3$ \\
\hline Equivalent width $(\mathrm{eV})$ & $110_{-60}^{+80}$ & $120 \pm 100$ & $<50$ \\
\hline
\end{tabular}

\section{DISCUSSION AND CONCLUSIONS}

We have reported results based on NUSTAR observations of the three absorbed radio galaxies NGC 612, 4C 73.08 and 3C 452, selected as possible CT candidates among the sample of P16. We derived constraints on their spectral parameters, first and foremost the absorbing column density $N_{\mathrm{H}}$. None of these sources is found to be formally Compton-thick, i.e. $N_{\mathrm{H}}<1.5 \times 10^{24} \mathrm{~cm}^{-2}$ in all three. The most absorbed one is NGC 612, for which we derived $N_{\mathrm{H}} \simeq 1 \times 10^{24} \mathrm{~cm}^{-2}$.

In all cases, we found no evidence for a strong Compton reflection component, neither in the approximation of reflection off a slab of infinite optical depth, nor assuming a more physical torus model. Moreover, the $\mathrm{Fe} \mathrm{K} \alpha$ line at $6.4 \mathrm{keV}$ is weak (see Table 4). These results are consistent with what is generally found in radio galaxies, which show weaker X-ray reprocessed features than Seyferts (e.g. Wozniak et al. 1998; Eracleous et al. 2000; Grandi 2001). In the past few years, NUSTAR provided us interesting results on single radio sources. For example, the NUSTAR spectra of the broad-line (i.e. type 1) radio galaxies 3C 382 and 3C 390.3 show a weak reflection component (Ballantyne et al. 2014; Lohfink et al. 2015), and Centaurus A (which is optically a type 2) shows no evidence for reflection at all (Fürst et al. 2016). However, the NuSTAR spectrum of Cygnus A (also a type 2) is consistent with significant reflection from Compton-thick matter, although the absorption along the line of sight is Compton-thin (Reynolds et al. 2015). The NUSTAR spectrum of the radio-loud quasar 4C 74.26 is also consistent with ionized reflection (Lohfink et al. 2017). In general, there can be different explanations for the weak reflection from the accretion disc (which would be expected if the disc is optically thick). In particular, the X-ray corona cold be outflowing, so that its radiation is beamed away from the accretion disc and the surrounding material (e.g. Beloborodov 1999; Malzac et al. 2001). This scenario could be promising for jetted sources, where the corona could be the base of the jet itself (e.g. Lohfink et al. 2013; Fabian et al. 2014). Another possibility is that the inner region of the accretion disc is optically thin, like an advection-dominated accretion flow (e.g. Narayan \& Yi 1994). Moreover, if we interpret the absorption in our sources as being due to a pc-scale torus, the accompanying reflection component is small (if any). This might suggest that the torus has a small covering factor, consistently with the spectral modelling with мүтоRus. The equivalent width of the $\mathrm{Fe} \mathrm{K} \alpha$ line alone provides a less model-dependent diagnostics. Following the detailed calculations by Yaqoob et al. (2001), the expected equivalent width (EW) of the line can be expressed as:

$\mathrm{EW} \simeq 40\left(\frac{f_{c}}{0.35}\right)\left(\frac{N_{\mathrm{H}}}{10^{23} \mathrm{~cm}^{-2}}\right) \mathrm{eV}$

where $f_{c}$ is the covering factor of the line-emitting material (spherically distributed), assuming solar $\mathrm{Fe}$ abundance and an incident power law with $\Gamma=1.65$. In the case of NGC 612 , for example, 
we derive a covering factor $f_{c}<0.17$ from the constraints on the Fe $\mathrm{K} \alpha$ line.

The apparent lack of reflection in the NUSTAR spectrum of 3C 452 is at odds with the results of Fioretti et al. (2013), who reported a reflection-dominated spectrum based on the 2007 Suzaku data. On the other hand, the absorbing column density is consistent between the two observations. We note that the $2-10 \mathrm{keV}$ flux was found to be $1.85 \times 10^{-12} \mathrm{erg} \mathrm{cm}^{-2} \mathrm{~s}^{-1}$ in the 2007 Suzaku observation (Fioretti et al. 2013), while we measured $3.4 \times 10^{-12}$ erg cm $\mathrm{cm}^{-2} \mathrm{~s}^{-1}$ in the $2017 \mathrm{NUSTAR}$ observation. This difference of a factor of almost 2, combined with the similar absorption, indicates an intrinsic luminosity variation, the source being possibly fainter and reflection-dominated during the 2007 Suzaku exposure. This behaviour might resemble that of the so-called 'changing-look' CT AGNs, namely sources undergoing changes from Compton-thin to reflection-dominated states (and vice-versa) likely due to dramatic variations of the nucleus luminosity (e.g. Matt et al. 2003, and references therein). On the other hand, we might have expected a stronger flux variation in $3 \mathrm{C} 452$ associated to this 'change of look'. Further observations with NuSTAR (which provides a much higher signal-to-noise above $10 \mathrm{keV}$ compared with Suzaku) would be needed to confirm this puzzling behaviour.

Concerning the scattered fraction, we derived a value of 0.20.3 per cent for NGC 612, around 2 per cent for $4 \mathrm{C} 73.08$, and around 9 per cent for $3 \mathrm{C} 452$. These values are reduced by a factor of 2 when assuming a more physical torus model. They are also roughly in agreement with previous estimates on NGC $612(0.55$ per cent according to Eguchi et al. 2011) and 4C 73.08 (from the best-fitting parameters of Evans et al. 2008), whereas for 3C 452 Fioretti et al. (2013) reported only an upper limit of 0.5 per cent. As we noted above, this discrepancy can be simply due to the contamination of the NUSTAR spectrum by the diffuse emission at energies lower than $5 \mathrm{keV}$. The scattered fraction $f_{s}$ could be related with the geometry of the torus. Indeed, a small value of $f_{s}$ (say below 0.5 per cent) might indicate a "hidden" or "buried" AGN, surrounded by a very geometrically thick torus (Ueda et al. 2007; Winter et al. 2009). However, this is at odds with the lack of strong reflection by the torus itself. It would be probably more reasonable to infer a small amount of scattering material. Furthermore, other interpretations are possible. For example, rather than Compton-thin scattering, the "soft excess" could be due to photoionized mission (Guainazzi \& Bianchi 2007). To test this scenario, high-resolution soft X-ray observations would be needed.

From the 2-10 keV luminosities that we derived, we can estimate the Eddington ratio of the sources. We assume for the luminosity the values found with the MYTORUs model, which takes into account Compton scattering. NGC 612 has an estimated black hole mass of around $4 \times 10^{8}$ solar masses (from the stellar velocity dispersion; Bettoni et al. 2003), and 3C 452 has a similar black hole mass of around $3 \times 10^{8}$ solar masses (from the host bulge magnitude; Marchesini et al. 2004). We are not aware of estimates of the black hole mass of $4 \mathrm{C} 73.08$. Using the $2-10 \mathrm{keV}$ bolometric corrections of Marconi et al. (2004), we estimate the bolometric luminosities to be $\sim 1.3 \times 10^{45} \mathrm{erg} \mathrm{s}^{-1}$ for NGC 612 and $\sim 1.6 \times 10^{46}$ $\mathrm{erg} \mathrm{s}^{-1}$ for $3 \mathrm{C} 452$. Given the black hole masses, the Eddington ratios are of the order of 0.02 for NGC 612 and 0.37 for 3C 452, respectively. 3C 452 is thus in a high-luminosity, strongly accreting state. NGC 612 is an order of magnitude less luminous and weaker in terms of accretion rate, but can still be considered a highluminosity and highly accreting AGN (e.g. Panessa et al. 2006; Kollmeier et al. 2006).

\subsection{CT radio galaxies}

According to our results, the constraints on the column density indicate that the three sources that we considered are absorbed but not $\mathrm{CT}$, consistently with the lack of strong reflection. We now examine the radio galaxies that have been reported as CT in the literature, and already mentioned in Sect. 1.

\subsubsection{Compact sources}

Mrk $668(\mathrm{OQ}+208)$ is a dust-obscured galaxy (DOG; Hwang \& Geller 2013; Hwang et al. 2013) hosting a compact, GigahertzPeaked Spectrum (GPS) radio source. This AGN has been reported as the first CT broad-line radio galaxy, from the analysis of XMM-Newton data (Guainazzi et al. 2004). Indeed, the hard Xray spectrum was found to be flat and showed a prominent neutral $\mathrm{Fe} \mathrm{K} \alpha$ line with equivalent width of $600 \mathrm{eV}$, suggesting a reflection-dominated spectrum, while the absorbing column density was found to be larger than $9 \times 10^{23} \mathrm{~cm}^{-2}$ (Guainazzi et al. 2004).

PKS $1607+26$ is also a GPS, labelled as a compact symmetric object (CSO). From XMM-Newton data, Tengstrand et al. (2009) suggested that this source could be CT $\left(N_{\mathrm{H}}>6 \times 10^{23} \mathrm{~cm}^{-2}\right)$. From Chandra data, Siemiginowska et al. (2016) later showed that the source is accompanied by a close, secondary X-ray source of uncertain nature, that XMM-Newton is not capable to resolve. Moreover, Siemiginowska et al. (2016) found that the Chandra spectrum is consistent with being essentially unobscured. This might suggest a changing-look scenario, but the presence of the secondary source complicates the interpretation and different scenarios are possible (see Siemiginowska et al. 2016).

TXS 2021+614 is another GPS/CSO. The Chandra spectrum is found to be flat $(\Gamma \simeq 0.8)$, and is consistent with absorption by $N_{\mathrm{H}} \gtrsim 9.5 \times 10^{23} \mathrm{~cm}^{-2}$ plus reflection (Siemiginowska et al. 2016). However, also given the lack of a significant $\mathrm{Fe} \mathrm{K} \alpha$ line, an alternative explanation based on non-thermal X-ray lobe emission cannot be ruled out (Siemiginowska et al. 2016).

Incidentally, we note that GPS/CSOs are young sources which could represent an early stage of the evolution of radio galaxies. Their X-ray absorption is not necessarily due to a torus, especially if the X-ray emission is produced by jets and/or lobes, which would rather be absorbed at galactic scales (e.g. Ostorero et al. 2016; Siemiginowska et al. 2016, see also Sect. 4.2).

\subsubsection{Extended sources}

Apart from the three compact radio galaxies mentioned above, the situation is even less clear for extended radio galaxies, and only a few of them have been proposed as CT candidates. Among the local objects, these are 3C 284, 3C 223 and 3C 321, in addition to the three sources examined in this paper.

3C 284 is a FRII radio source, hosted by a disturbed elliptical with dust lanes (Floyd et al. 2008). Croston et al. (2004) reported an X-ray absorbing column density $N_{\mathrm{H}}=2.6_{-1.0}^{+1.4} \times 10^{23} \mathrm{~cm}^{-2}$ from the analysis of XMM-Newton data. However, Hardcastle et al. (2006) reported $N_{\mathrm{H}}>1.2 \times 10^{24}$ from the same data set, ascribing the discrepancy to changes in the XMM-Newton calibration files. A similar column density $\left(N_{\mathrm{H}}=2.0_{-0.7}^{+1.4} \times 10^{24} \mathrm{~cm}^{-2}\right)$ was reported by Corral et al. (2014), from the same XMM-Newton data.

3C 223 is another FRII radio galaxy, for which Croston et al. (2004) reported a column density smaller than $1 \times 10^{23} \mathrm{~cm}^{-2}$ based on XMM-Newton data. Hardcastle et al. (2006) found $N_{\mathrm{H}}=$ 
$5.7_{-36}^{+7.8} \times 10^{23} \mathrm{~cm}^{-2}$ from their re-analysis of the same data. Corral et al. (2014) instead suggested that the XMM-Newton spectrum is reflection-dominated, estimating a column density larger than $10^{24} \mathrm{~cm}^{-2}$. Finally, from the same data set LaMassa et al. (2014) suggested that the source is surrounded by a globally Comptonthin medium $\left(N_{\mathrm{H}} \sim 10^{23} \mathrm{~cm}^{-2}\right)$, giving rise to the reflection component, while the line-of-sight column density is Compton-thick $\left(N_{\mathrm{H}}>1.67 \times 10^{24} \mathrm{~cm}^{-2}\right)$. This would be a rather unique case among absorbed AGNs.

3C 321 is a FRII radio galaxy with a peculiar radio morphology, and in the process of merging with a companion galaxy, which might also host an active nucleus (Evans et al. 2008). Moreover, a dust lane is seen at kpc scales (e.g. Martel et al. 1999). From the analysis of Chandra data, Evans et al. (2008) found the nucleus of $3 \mathrm{C} 321$ to be obscured by a column density $\sim 10^{24} \mathrm{~cm}^{-2}$. However, at least part of this obscuration could be due to the complex galaxy environment.

Finally, Wilkes et al. (2013) reported eight CT candidates among the high-redshift $(1<z<2)$ sources of the 3CRR catalogue (Laing et al. 1983), from Chandra data. Given the low number of counts, this suggestion is not based on spectral fits, but mostly on the X-ray hardness ratio, on the $\mathrm{X}$-ray to radio luminosity ratio, and on the $\left[\mathrm{O}_{\text {III }}\right] \lambda 5007$ emission line. The estimated column densities for these eight candidates are $\sim 2 \times 10^{24} \mathrm{~cm}^{-2}$, but the faintness of the sources makes this result quite uncertain (see the discussion in Wilkes et al. 2013).

Taken together, these findings indicate that a handful of radio galaxies could be consistent with being heavily absorbed. On the other hand, the uncertainties are large, also because of the lack of high-quality data at high energies. As a result, we currently lack clear-cut evidences for formally $\mathrm{CT}$ radio galaxies, i.e. with $N_{\mathrm{H}}$ well constrained to be larger than $1.5 \times 10^{24} \mathrm{~cm}^{-2}$. Of course this threshold is somewhat arbitrary, but it does have a physical meaning, namely the absorbing material becomes optically thick to Compton scattering. In any case, in radio galaxies we seem to have an indication of rare (or even lacking) heavy absorption, i.e. exceeding a few times $10^{24} \mathrm{~cm}^{-2}$, compared with radio-quiet Seyferts (see also P16).

Apart from the few single sources discussed above, from P16 and the present work we have no evidence for heavily absorbed sources among the hard X-ray selected sample of Bassani et al. (2016). An obvious selection bias against CT sources is absorption itself, which can produce a strong reduction of flux. Even at hard Xrays, a CT column density implies significant Compton downscattering to energies where photoelectric absorption dominates (e.g. Matt et al. 1999). Therefore, even hard X-ray surveys are not totally immune from the absorption bias (e.g. Malizia et al. 2009; Burlon et al. 2011; Malizia et al. 2012). However, even if the numbers are not large enough to draw definitive conclusions, we have a tentative indication that only a few radio galaxies (if any) are CT. This might hint for a difference between the absorption properties of radio galaxies and radio-quiet Seyferts, which deserves further investigation.

\subsection{What is the nature of the absorber?}

The neutral absorption seen in radio galaxies, just like radio-quiet Seyferts, is generally ascribed to a putative pc-scale torus (e.g. Urry \& Padovani 1995; Hardcastle et al. 2009). The presence of a molecular, dusty torus in Seyferts is also widely invoked to explain the infrared (IR) emission, which is often interpreted as thermal emission from hot dust (e.g. Pier \& Krolik 1993). In recent years, optical/IR interferometry permitted to resolve the pc-scale environment of AGNs and directly observe the torus (e.g. Burtscher et al. 2013, and references therein). The basic tenet of a torusdominated IR emission has been challenged by the observation of strong mid-IR emission originating from the polar region at 10 100 pc-scales, rather than the torus (e.g. Hönig et al. 2013; Asmus et al. 2016). On the other hand, the near-IR emission is likely to originate from the equatorial plane (e.g. Hönig et al. 2013). A two-component disc+outflow scenario is thus emerging, where dust clouds are accreted in the disc plane and are in part lifted up by radiation pressure, forming an outflow cone (Hönig \& Kishimoto 2017). Future, multiwavelength observations on radio galaxies will be needed to gain further insight on the relation between the mechanisms of accretion (disc/torus) and ejection (jet/outflows). Interestingly, NGC 612, namely the most absorbed source reported in P16 and discussed here, does not show evidence for any mid-IR emission from the nucleus, being dominated by star formation at much larger scales (Asmus et al. 2014; Duah Asabere et al. 2016). This points to a relatively weak AGN, in agreement with the low Eddington ratio (0.02) that we have estimated. Moreover, given the peculiar morphology of this source, we may also speculate that the $\mathrm{X}$-ray absorption is (at least partly) due to a large-scale structure rather than to a pc-scale torus.

In general, it is possible that at least part of the absorption is due to different material than the torus, eventually located further away and even at galactic scales. For example, the presence of dust lanes is ubiquitous in Seyferts (e.g. Malkan et al. 1998) and it is likely related with Compton-thin X-ray obscuration (Matt 2000; Guainazzi et al. 2005). Furthermore, $21 \mathrm{~cm} \mathrm{H}$ I absorption is commonly observed in radio AGNs and it can trace rotating discs, outflows or more complex morphologies (e.g. Morganti et al. 2001; Geréb et al. 2015). Recently, Glowacki et al. (2017) and Moss et al. (2017) reported a correlation between X-ray absorption and $21 \mathrm{~cm}$ absorption in a sample of obscured radio AGNs. Such a correlation was also found by Ostorero et al. $(2010,2016)$ for GPS sources, suggesting that the $\mathrm{X}$-ray obscuration might be caused by gas at large scales (Siemiginowska et al. 2016). It remains to be seen whether the $21 \mathrm{~cm}$ absorption is due to a pc-scale torus, or to a galactic inflow at a few tens of pc-scale, or to a kpc-wide galactic structure (Moss et al. 2017). Interestingly, old, extended radio sources seem to show a lower fraction of $21 \mathrm{~cm} \mathrm{H}$ i detection than young, compact ones (Chandola et al. 2013). Then, if the H I and Xray absorption are truly related, we expect to observe weaker X-ray absorption in extended radio sources. Among the sources discussed in this paper and in Sect. 4.1.2, $\mathrm{H}$ I absorption has been detected in NGC 612 (Emonts et al. 2008, see also Sect. 1), in 3C 452 (Gupta \& Saikia 2006; Chandola et al. 2013) and in 3C 321 (Chandola et al. 2012). The column density is estimated to be $N_{\mathrm{H}_{\mathrm{I}}} \simeq 5 \times 10^{21}$ $\mathrm{cm}^{-2}$ for NGC 612 (Emonts et al. 2008), $N_{\mathrm{H}_{\mathrm{I}}} \simeq 6.4 \times 10^{20} \mathrm{~cm}^{-2}$ for 3C 452 (Gupta \& Saikia 2006) and $N_{\mathrm{H}_{\mathrm{I}}} \simeq 9.2 \times 10^{21} \mathrm{~cm}^{-2}$ for 3C 321 (Chandola et al. 2012). $N_{\mathrm{H}_{\mathrm{I}}}$ seems thus to be much smaller than the column density measured in X-rays $\left(N_{\mathrm{H}} \sim 10^{23-24} \mathrm{~cm}^{-2}\right)$. However, the inferred $N_{\mathrm{H}_{\mathrm{I}}}$ is generally considered a lower limit, because of a degeneracy with the (unknown) spin temperature and covering fraction (Gupta \& Saikia 2006; Geréb et al. 2015). Owing to such uncertainties, we cannot rule out a significant contribution to the X-ray column density by atomic hydrogen seen at radio frequencies, and that could reside at distances larger than the pc-scale torus. 


\section{SUMMARY}

We have studied the X-ray spectra of three CT candidates (NGC 612, 4C 73.08 and 3C 452) among the hard X-ray selected radio galaxies investigated in P16, making use of NUSTAR, XMMNewton, Swift/BAT and INTEGRAL data. Our conclusions can be summarized as follows:

- We do not find formally CT sources. NGC 612 is the only source consistent with a column density of around $1 \times 10^{24} \mathrm{~cm}^{-2}$. No source shows evidences for a strong reflection component. Comparing with a past Suzaku observation, it is possible that 3C 452 switched from a reflection-dominated to a Compton-thin state over the course of 10 years.

- The lack of strong evidences for CT hard X-ray selected radio galaxies, also coupled with residual uncertainties on the real nature of the few CT radio galaxies reported in the literature, is quite puzzling. Although we cannot rule out the effect of a selection bias, this result could hint for a discrepancy between the average absorption properties of radio-loud and radio-quiet AGNs.

- The origin of the X-ray absorbing medium is not obvious. A significant role could be played by material different from the classical pc-scale torus, such as that traced by $21 \mathrm{~cm} \mathrm{Hi}$ absorption, which can be located much farther away (e.g. at galactic scales for NGC 612).

\section{ACKNOWLEDGEMENTS}

We thank the referee for helpful comments that improved the paper.

We acknowledge the use of public data from the NUSTAR, XMM-Newton, Swift and INTEGRAL data archives. This research has made use of data, software and/or web tools obtained from NASA's High Energy Astrophysics Science Archive Research Center (HEASARC), a service of Goddard Space Flight Center and the Smithsonian Astrophysical Observatory, and of the NuSTAR Data Analysis Software jointly developed by the ASI Space Science Data Center (SSDC, Italy) and the California Institute of Technology (USA). We acknowledge financial support from ASI under contract ASI/INAF 2013-025-R01.

\section{REFERENCES}

Anders E., Grevesse N., 1989, Geochimica Cosmochimica Acta, 53, 197 Antonucci R., 1993, ARA\&A, 31, 473

Antonucci R. R. J., Miller J. S., 1985, ApJ, 297, 621

Arnaud K. A., 1996, in Jacoby G. H., Barnes J., eds, Astronomical Society of the Pacific Conference Series Vol. 101, Astronomical Data Analysis Software and Systems V. p. 17

Asmus D., Hönig S. F., Gandhi P., Smette A., Duschl W. J., 2014, MNRAS, 439, 1648

Asmus D., Hönig S. F., Gandhi P., 2016, ApJ, 822, 109

Ballantyne D. R., et al., 2014, ApJ, 794, 62

Baloković M., et al., 2014, ApJ, 794, 111

Bassani L., Dadina M., Maiolino R., Salvati M., Risaliti G., Della Ceca R., Matt G., Zamorani G., 1999, ApJS, 121, 473

Bassani L., Venturi T., Molina M., Malizia A., Dallacasa D., Panessa F., Bazzano A., Ubertini P., 2016, MNRAS, 461, 3165

Baumgartner W. H., Tueller J., Markwardt C. B., Skinner G. K., Barthelmy S., Mushotzky R. F., Evans P. A., Gehrels N., 2013, ApJS, 207, 19

Beloborodov A. M., 1999, ApJ, 510, L123

Bettoni D., Falomo R., Fasano G., Govoni F., 2003, A\&A, 399, 869

Bird A. J., et al., 2010, ApJS, 186, 1

Black A. R. S., Baum S. A., Leahy J. P., Perley R. A., Riley J. M., Scheuer P. A. G., 1992, MNRAS, 256, 186
Brightman M., Nandra K., Salvato M., Hsu L.-T., Aird J., Rangel C., 2014, MNRAS, 443, 1999

Burlon D., Ajello M., Greiner J., Comastri A., Merloni A., Gehrels N., 2011, ApJ, 728, 58

Burtscher L., et al., 2013, A\&A, 558, A149

Chandola Y., Sirothia S. K., Saikia D. J., Gupta N., 2012, Bulletin of the Astronomical Society of India, 40, 139

Chandola Y., Gupta N., Saikia D. J., 2013, MNRAS, 429, 2380

Corral A., et al., 2014, A\&A, 569, A71

Croston J. H., Birkinshaw M., Hardcastle M. J., Worrall D. M., 2004, MNRAS, 353,879

Duah Asabere B., Horellou C., Jarrett T. H., Winkler H., 2016, A\&A, 592, A20

Eguchi S., Ueda Y., Terashima Y., Mushotzky R., Tueller J., 2009, ApJ, 696, 1657

Eguchi S., Ueda Y., Awaki H., Aird J., Terashima Y., Mushotzky R., 2011, ApJ, 729, 31

Ekers R. D., Goss W. M., Kotanyi C. G., Skellern D. J., 1978, A\&A, 69, L21

Elitzur M., Shlosman I., 2006, ApJ, 648, L101

Emonts B. H. C., Morganti R., Oosterloo T. A., Holt J., Tadhunter C. N., van der Hulst J. M., Ojha R., Sadler E. M., 2008, MNRAS, 387, 197

Eracleous M., Sambruna R., Mushotzky R. F., 2000, ApJ, 537, 654

Evans D. A., Worrall D. M., Hardcastle M. J., Kraft R. P., Birkinshaw M., 2006, ApJ, 642, 96

Evans D. A., et al., 2008, ApJ, 675, 1057

Fabian A. C., Iwasawa K., 1999, MNRAS, 303, L34

Fabian A. C., Parker M. L., Wilkins D. R., Miller J. M., Kara E., Reynolds C. S., Dauser T., 2014, MNRAS, 439, 2307

Fioretti V., Angelini L., Mushotzky R. F., Koss M., Malaguti G., 2013, A\&A, 555, A44

Floyd D. J. E., et al., 2008, ApJS, 177, 148

Fürst F., et al., 2016, ApJ, 819, 150

Geréb K., Maccagni F. M., Morganti R., Oosterloo T. A., 2015, A\&A, 575, A44

Gilli R., Comastri A., Hasinger G., 2007, A\&A, 463, 79

Glowacki M., et al., 2017, MNRAS, 467, 2766

Gopal-Krishna Wiita P. J., 2000, A\&A, 363, 507

Grandi P., 2001, Mem. Soc. Astron. Italiana, 72, 91

Guainazzi M., Bianchi S., 2007, MNRAS, 374, 1290

Guainazzi M., Siemiginowska A., Rodriguez-Pascual P., Stanghellini C., 2004, A\&A, 421, 461

Guainazzi M., Matt G., Perola G. C., 2005, A\&A, 444, 119

Gupta N., Saikia D. J., 2006, MNRAS, 370, L80

Hardcastle M. J., Evans D. A., Croston J. H., 2006, MNRAS, 370, 1893

Hardcastle M. J., Evans D. A., Croston J. H., 2009, MNRAS, 396, 1929

Harrison F. A., et al., 2013, ApJ, 770, 103

Hewitt A., Burbidge G., 1991, ApJS, 75, 297

Holt J., Tadhunter C. N., González Delgado R. M., Inskip K. J., Rodriguez Zaurin J., Emonts B. H. C., Morganti R., Wills K. A., 2007, MNRAS, 381,611

Hönig S. F., Kishimoto M., 2017, ApJ, 838, L20

Hönig S. F., et al., 2013, ApJ, 771, 87

Hwang H. S., Geller M. J., 2013, ApJ, 769, 116

Hwang H. S., Andrews S. M., Geller M. J., 2013, ApJ, 777, 38

Isobe N., Tashiro M., Makishima K., Iyomoto N., Suzuki M., Murakami M. M., Mori M., Abe K., 2002, ApJ, 580, L111

Jansen F., et al., 2001, A\&A, 365, L1

Kalberla P. M. W., Burton W. B., Hartmann D., Arnal E. M., Bajaja E., Morras R., Pöppel W. G. L., 2005, A\&A, 440, 775

Kollmeier J. A., et al., 2006, ApJ, 648, 128

La Franca F., et al., 2005, ApJ, 635, 864

LaMassa S. M., Yaqoob T., Ptak A. F., Jia J., Heckman T. M., Gandhi P., Meg Urry C., 2014, ApJ, 787, 61

Laing R. A., Riley J. M., Longair M. S., 1983, MNRAS, 204, 151

Lansbury G. B., et al., 2017, ApJ, 836, 99

Lanzuisi G., et al., 2015, A\&A, 573, A137 
Lara L., Cotton W. D., Feretti L., Giovannini G., Marcaide J. M., Márquez I., Venturi T., 2001, A\&A, 370, 409

Lohfink A. M., et al., 2013, ApJ, 772, 83

Lohfink A. M., et al., 2015, ApJ, 814, 24

Lohfink A. M., et al., 2017, ApJ, 841, 80

Madsen K. K., Beardmore A. P., Forster K., Guainazzi M., Marshall H. L., Miller E. D., Page K. L., Stuhlinger M., 2017, AJ, 153, 2

Magdziarz P., Zdziarski A. A., 1995, MNRAS, 273, 837

Malizia A., Stephen J. B., Bassani L., Bird A. J., Panessa F., Ubertini P., 2009, MNRAS, 399, 944

Malizia A., Bassani L., Bazzano A., Bird A. J., Masetti N., Panessa F., Stephen J. B., Ubertini P., 2012, MNRAS, 426, 1750

Malkan M. A., Gorjian V., Tam R., 1998, ApJS, 117, 25

Malzac J., Beloborodov A. M., Poutanen J., 2001, MNRAS, 326, 417

Marchesini D., Celotti A., Ferrarese L., 2004, MNRAS, 351, 733

Marconi A., Risaliti G., Gilli R., Hunt L. K., Maiolino R., Salvati M., 2004, MNRAS, 351, 169

Martel A. R., et al., 1999, ApJS, 122, 81

Mateos S., et al., 2017, ApJ, 841, L18

Matt G., 2000, A\&A, 355, L31

Matt G., Brandt W. N., Fabian A. C., 1996, MNRAS, 280, 823

Matt G., Pompilio F., La Franca F., 1999, New Astron., 4, 191

Matt G., Fabian A. C., Guainazzi M., Iwasawa K., Bassani L., Malaguti G., 2000, MNRAS, 318, 173

Matt G., Guainazzi M., Maiolino R., 2003, MNRAS, 342, 422

Molina M., Bassani L., Malizia A., Stephen J. B., Bird A. J., Bazzano A., Ubertini P., 2013, MNRAS, 433, 1687

Morganti R., Oosterloo T. A., Tadhunter C. N., van Moorsel G., Killeen N., Wills K. A., 2001, MNRAS, 323, 331

Moss V. A., et al., 2017, preprint, (arXiv: 1707.01542)

Murphy K. D., Yaqoob T., 2009, MNRAS, 397, 1549

Narayan R., Yi I., 1994, ApJ, 428, L13

Ostorero L., et al., 2010, ApJ, 715, 1071

Ostorero L., Morganti R., Diaferio A., Siemiginowska A., Stawarz Ł., Moderski R., Labiano A., 2016, Astronomische Nachrichten, 337, 148

Panessa F., Bassani L., Cappi M., Dadina M., Barcons X., Carrera F. J., Ho L. C., Iwasawa K., 2006, A\&A, 455, 173

Panessa F., et al., 2016, MNRAS, 461, 3153

Parisi P., et al., 2009, A\&A, 507, 1345

Parisi P., et al., 2014, A\&A, 561, A67

Piconcelli E., Jimenez-Bailón E., Guainazzi M., Schartel N., RodríguezPascual P. M., Santos-Lleó M., 2004, MNRAS, 351, 161

Pier E. A., Krolik J. H., 1993, ApJ, 418, 673

Reynolds C. S., et al., 2015, ApJ, 808, 154

Ricci C., Ueda Y., Koss M. J., Trakhtenbrot B., Bauer F. E., Gandhi P., 2015, ApJ, 815, L13

Risaliti G., Maiolino R., Salvati M., 1999, ApJ, 522, 157

Risaliti G., Elvis M., Gilli R., 2002, ApJ, 566, L67

Sambruna R. M., Eracleous M., Mushotzky R. F., 1999, ApJ, 526, 60

Sazonov S., Revnivtsev M., Burenin R., Churazov E., Sunyaev R., Forman W. R., Murray S. S., 2008, A\&A, 487, 509

Sazonov S., Churazov E., Krivonos R., 2015, MNRAS, 454, 1202

Severgnini P., Caccianiga A., Della Ceca R., 2012, A\&A, 542, A46

Shelton D. L., Hardcastle M. J., Croston J. H., 2011, Monthly Notices of the Royal Astronomical Society, 418, 811

Siemiginowska A., Sobolewska M., Migliori G., Guainazzi M., Hardcastle M., Ostorero L., Stawarz Ł., 2016, ApJ, 823, 57

Strom R. G., Chen R., Yang J., Peng B., 2013, MNRAS, 430, 2090

Tengstrand O., Guainazzi M., Siemiginowska A., Fonseca Bonilla N., Labiano A., Worrall D. M., Grandi P., Piconcelli E., 2009, A\&A, 501, 89

Turner T. J., George I. M., Nandra K., Mushotzky R. F., 1997, ApJS, 113, 23

Ubertini P., et al., 2003, A\&A, 411, L131

Ueda Y., Akiyama M., Ohta K., Miyaji T., 2003, ApJ, 598, 886

Ueda Y., et al., 2007, ApJ, 664, L79

Urry C. M., Padovani P., 1995, PASP, 107, 803

Verner D. A., Ferland G. J., Korista K. T., Yakovlev D. G., 1996, ApJ, 465, 487
Véron-Cetty M. P., Véron P., 2001, A\&A, 375, 791

Véron-Cetty M.-P., Véron P., 2006, A\&A, 455, 773

Wilkes B. J., et al., 2013, ApJ, 773, 15

Winter L. M., Mushotzky R. F., Reynolds C. S., Tueller J., 2009, ApJ, 690, 1322

Wozniak P. R., Zdziarski A. A., Smith D., Madejski G. M., Johnson W. N., 1998, MNRAS, 299, 449

Yaqoob T., 2012, MNRAS, 423, 3360

Yaqoob T., George I. M., Nandra K., Turner T. J., Serlemitsos P. J., Mushotzky R. F., 2001, ApJ, 546, 759

Zdziarski A. A., Johnson W. N., Magdziarz P., 1996, MNRAS, 283, 193

Życki P. T., Done C., Smith D. A., 1999, MNRAS, 309, 561

This paper has been typeset from a $\mathrm{T}_{\mathrm{E}} \mathrm{X} / \mathrm{L}_{\mathrm{E}} \mathrm{X}$ file prepared by the author. 